\title{
Quench Limit Model and Measurements for Steady State Heat Deposits in LHC Magnets
}

\author{
Dariusz Bocian, Bernd Dehning, and Andrzej Siemko
}

\begin{abstract}
A quench, transition of a conductor from the superconducting to the normal conducting state, occurs irreversibly in accelerator magnets if one of the three parameters: temperature, magnetic field or current density, exceeds its critical value. The protons lost from the beam and impacting on the vacuum chamber, create a secondary particle shower that deposes its energy in the magnet coil. Energy deposited in the superconductor by these particles can provoke quenches that can be detrimental for the accelerator operation. A network model is developed to study the thermodynamic behavior of the LHC magnets. The results of the heat flow simulation in the main dipole and quadrupole LHC magnets calculated by means of the network model were validated with measurements performed at superfluid helium temperatures in the CERN magnet test facility. A steady state heat flow was introduced in the magnet coil by using a dedicated internal heating apparatus (IHA) installed inside the magnet cold bore. The value of the heat source flux flow is determined from the network model. The magnet coil current, which is required to quench the magnet coil, is predicted accordingly.
\end{abstract}

Index Terms-Heat transfer, LHC superconducting magnets, quench level calculations, quench level measurements, quench limit, steady state heat deposits.

\section{INTRODUCTION}

$\mathbf{T}$ HE Large Hadron Collider (LHC) operation [1] requires a careful control of the beam losses around the LHC ring. The beam losses involving a significant number of particles, may have severe consequences for the accelerator equipment [2]. One of the operation issues of the LHC is related to the quenches induced in the superconducting (SC) magnets by the particles lost from the beams. In order to cope with this problem a control system has been developed to predict imminent beam induced quenches and dump the beam before quenching any of the main magnets. This system is based on beam loss monitors (BLM) [3] and is using beam dumps, collimators and beam absorbers. The BLM system is measuring the flux of secondary particles (hadronic shower), created by lost protons hitting the beam screen, the cold bore and the superconducting coils [4]. The activation threshold for the BLM system needs to be set comparing the energy deposition due to the hadronic shower to the expected quench level of the SC magnets [5]. The beam loss duration ranges from a few nano-seconds (transient) to several seconds (steady state), depending on the specific failure mode [6]. Steady state losses are mainly caused by the debris of the

Manuscript received August 27, 2008. First published June 05, 2009; current version published July 15,2009

D. Bocian is with CERN, Geneva, Switzerland. He is also with IFJ PAN, Krakow, Poland (e-mail: Dariusz.Bocian@cern.ch).

B. Dehning and A. Siemko are with CERN, Geneva, Switzerland.

Color versions of one or more of the figures in this paper are available online at http://ieeexplore.ieee.org.

Digital Object Identifier 10.1109/TASC.2009.2019060 proton-proton interactions at accelerator insertion regions, originating from the residual beam-gas interactions and from the interaction of lost protons with the collimators. The dominant loss locations are the insertion and collimation regions and the nearby dispersion suppressors [2]. The continuous energy deposition in the LHC main magnets due to the beam losses implies a continuous evacuation of the heat from the coil to keep the temperature in the superconducting cables below their critical temperatures. The heat flow in magnet coils at steady state regime is mainly limited by the size of the helium channels and the heat conduction of the cable insulation. The power dissipation in the superconducting magnet components leads to a complex process of the heat flow, but in many cases a simplified model for heat transfer is sufficient [7].

In this paper, the Network Model developed for the main LHC dipole and quadrupole superconducting magnets operating at $1.9 \mathrm{~K}$ is presented. The Section II is devoted to the Network Model construction and the way that superconducting cable structure is transformed into a thermal resistance network. In Section III, the basic assumptions of the simulations are discussed. The model validation with the measurements performed at superfluid helium is the subject of Section IV and is complementary to the results previously obtained at normal liquid helium temperatures [7]. The experimental setup is discussed and the results of the simulations and measurements and their relative differences for the case of two main LHC lattice magnets are shown in this section. The quench limits of the two main LHC magnet families operating at $1.9 \mathrm{~K}$ and affected by Gaussian and radial beam loss profile are also presented and discussed.

\section{NETWORK MODELS}

The network models are developed to study the thermodynamic behavior of the magnet coils and to calculate the quench levels of the LHC magnets for expected beam loss profiles. This paper discuses the case of the two families of the main LHC magnets operating at $1.9 \mathrm{~K}$, namely the main bending dipoles $\mathrm{MB}$ and the main focusing quadrupoles MQ.

The detailed description of the relevant network models and the first results of the heat flow simulations for the magnets operating at $4.5 \mathrm{~K}$ are presented in [7]. In this section, a short summary of the main features of the developed network models are presented. Fig. 1 shows schematically the construction of the 2-dimensional network models. The vital input information for the models is taken from technical drawings, which provide the details to create the mesh of thermal elements. Other required information is: the heat conductivity, magnetic field distribution, temperature margin, beam loss profiles and the model validation data.

The fundamental unit of the Network Model is the magnet coil cable. The cable equivalent network is depicted in Fig. 2. 


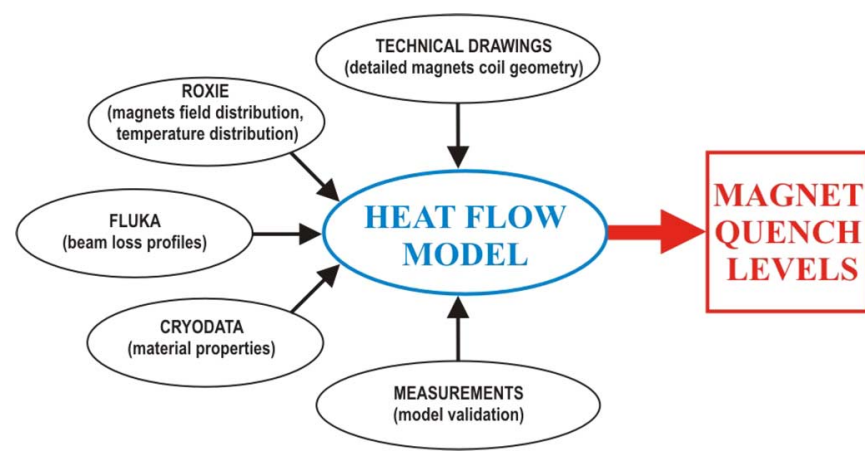

Fig. 1. Network Model construction overview.

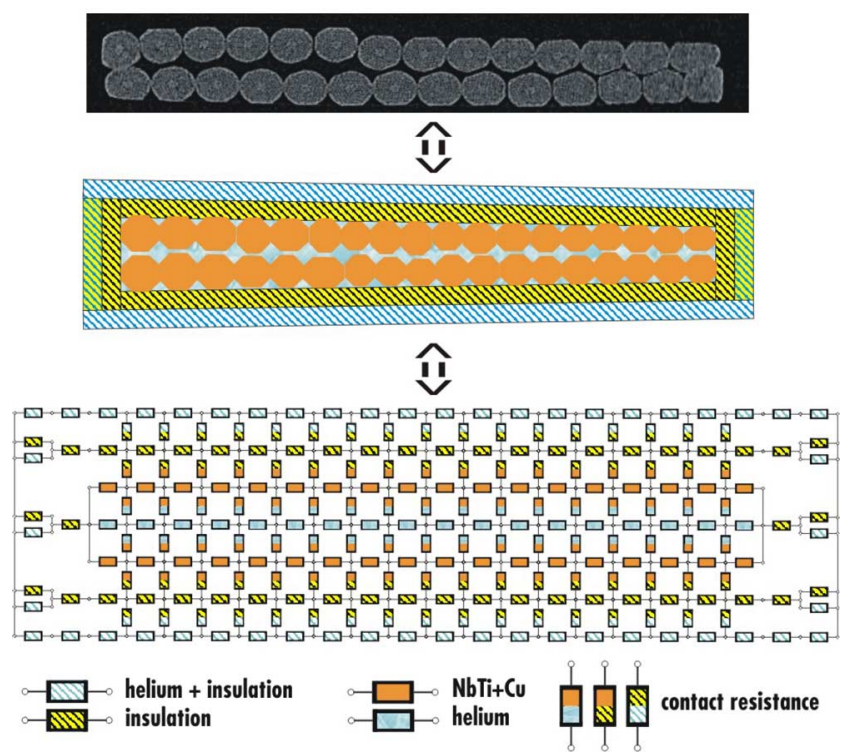

Fig. 2. Picture of the superconducting cable, its physical model and its equivalent network model.

The coil network model is constructed from the cable unit. The cable sample was normalized to 1 meter of cable length. Other coil elements i.e. ground insulation, inter-layer insulation, helium channel around the cold bore were included in the model with segmentation corresponding to the cable dimensions. The values of the thermal conductivity for the calculation of thermal resistance for each of the thermal resistors in the network model are taken from a commercially available database [10] and literature [11]-[13].

\section{NUMERICAL SimUlationS}

The recent Network Model consider of the seven different heat conduction mechanisms, namely the superfluid helium heat conductivity in narrow and wide channels, the nucleate boiling in the normal liquid helium, the convection and the heat conductivity of normal liquid helium and the convection and heat conductivity of gaseous helium. Fig. 3 shows schematically the implementation of the helium conduction mechanisms to the Network Model. The proper helium modeling in the network models of the magnets operating at $1.9 \mathrm{~K}$ is fundamental to understand the heat flow mechanism in the magnet coil. The bold (red) rectangle blocks indicate the most conservative heat evacuation path.

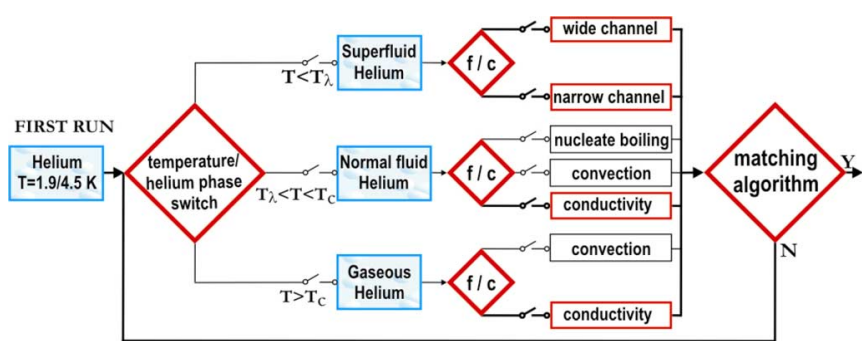

Fig. 3. Helium modeling in Network Model. The bold (red) rectangle blocks indicate the most conservative heat evacuation path. $\mathrm{f} / \mathrm{c}$ mean flux/channel size switch.

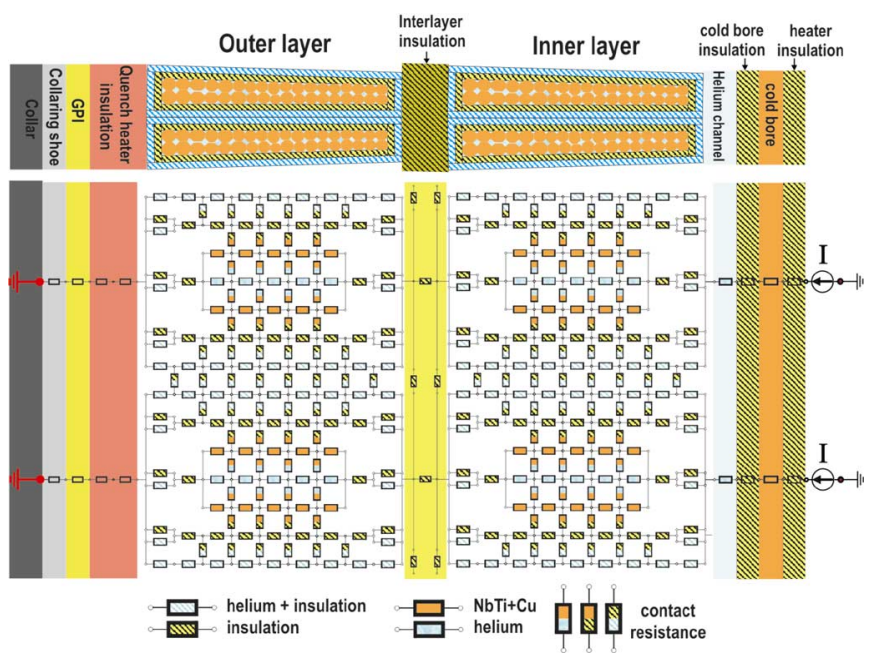

Fig. 4. Characteristic part of the superconducting coil network model. Internal Heating Apparatus heat source (I) is indicated at the right side of the picture.

Fig. 4 shows the implementation of the IHA to the network model. The volumes occupied by helium in the magnet coil are considered as narrow channels. In addition, these volumes are considered as semi-closed volumes, without "easy" direct link to the helium bath in the magnet cold mass. From the model sensitivity analysis, it was found that the main heat flow barriers are the cable insulation and the ground insulation flaps in the helium channel around the cold bore. In case of magnets operating at $4.5 \mathrm{~K}$, the steady heat load to the semi-closed volumes leads to the transition of helium from liquid to gaseous phase in the coil. The magnets operating in superfluid helium at $1.9 \mathrm{~K}$ are more complex. They can follow all seven mechanisms of heat flow presented in Fig. 3. The quench can occur not only at the gaseous phase of the helium in the magnet coil (SC critical temperatures above $4.5 \mathrm{~K}$ ) but also at the liquid helium regime, according to the $\mathrm{SC}$ critical temperature of the magnet. Moreover, in the $1.9 \mathrm{~K}$ models one can observe a case in which the helium temperature rises from superfluid $(1.9-2.16 \mathrm{~K}$ at 1.3 bar) to supercritical phase ( $4.5 \mathrm{~K}$ at $1.3 \mathrm{bar}$ ). This case does not correspond to the working regimes of the magnets (neither injection nor operation at nominal constant current). Nevertheless, it is worth to be discussed. Following rise the helium temperature one can observe the moment at which the heat flow scheme changes due to the blocking existing heat flow paths. One of the vital conclusions coming from the helium heat conduction studies is the fact that the heat conduction of the helium between $\lambda$-point and nucleate boiling phase is relatively poor and 


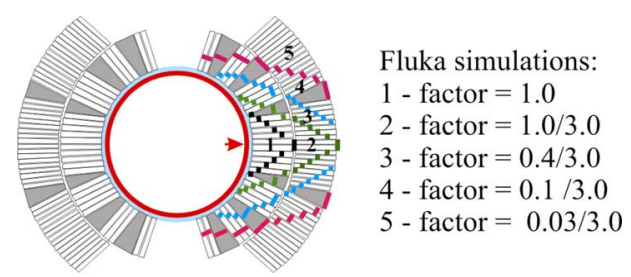

Fig. 5. Schematic diagram of the heat deposits in the magnet coil (left) and coefficients of the heat deposits in case of the Gaussian profile calculated by the FLUKA [8].
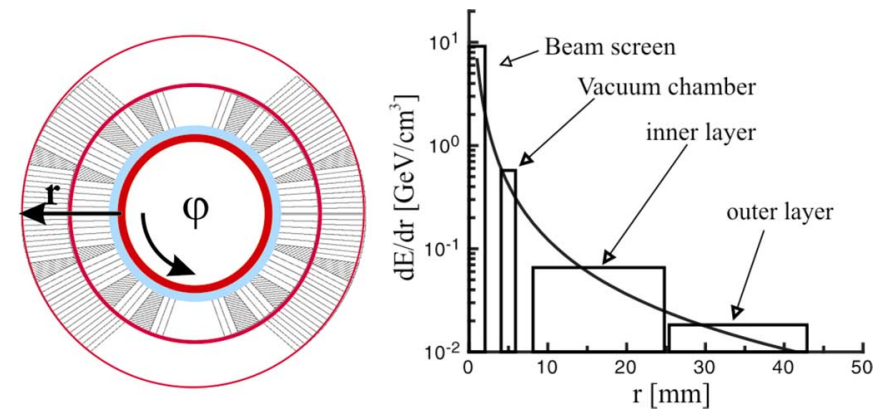

Fig. 6. Schematic diagram of the heat deposits in the magnet coil (left) and the radial energy density along the most exposed azimuth [9].

in practice negligible. Due to the narrow and semi-closed channels for temperatures around the $\lambda$-point, one gets the value of helium heat conduction only one order of magnitude above that of the polyimide insulation and coming to the same order (of $\left.10^{-2} \mathrm{~W} / \mathrm{m} \cdot \mathrm{K}\right)$ when reaching the temperatures a bit below nucleate boiling temperature $(4.5 \mathrm{~K}$ at $1.3 \mathrm{bar})$. All helium regimes presented above are included in the model.

The heat deposition profiles characteristic of the typical beam loss scenarios are shown in Fig. 5 [8] and Fig. 6 [9]. Both of them are expected during the LHC operation. The Gaussian beam losses are expected in the arc magnets whereas the radial beam losses are expected in the interaction, collimation and dispersion suppressor regions. In the simulations the Gaussian beam loss profile has been segmented to five sub-profiles and the weighting factors calculated with the FLUKA are indicated in Fig. 5.

\section{Model VALidATION AND Simulation Results}

A dedicated tool for heating the magnet coil from the inside of the cold bore has been developed and used during the experiments described in the paper. The Internal Heating Apparatus consists of $1 \mathrm{~m}$ long and $0.5 \mathrm{~mm}$ thick stainless steel tube and the insulated heating strips glued to this tube. The heating strips are made of $25 \mu \mathrm{m}$ thick stainless steel, insulated with $100 \mu \mathrm{m}$ thick polyimide film. An expanding cone system is installed inside the support tube to assure good thermal contact with the cold bore. The support tube has a $3 \mathrm{~mm}$ wide gap along its length to assure the flexibility of the tube during the installation and during forced expansion inside the cold bore. The heating strips are equipped with four wires to allow precise measurements of the strip voltages, necessary to calculate the power dissipated in the strips. Each of the four heating strips has been powered independently with one DC power supply. The temperature of the support tube has been controlled by means of the two PT100 temperature probes installed in the middle and

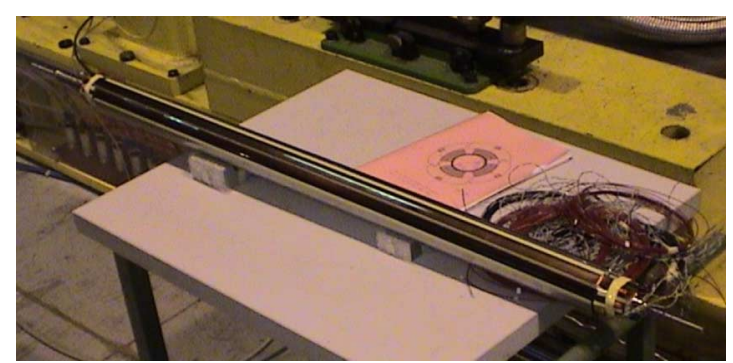

Fig. 7. Internal Heating Apparatus ready for installation inside the magnet cold bore.

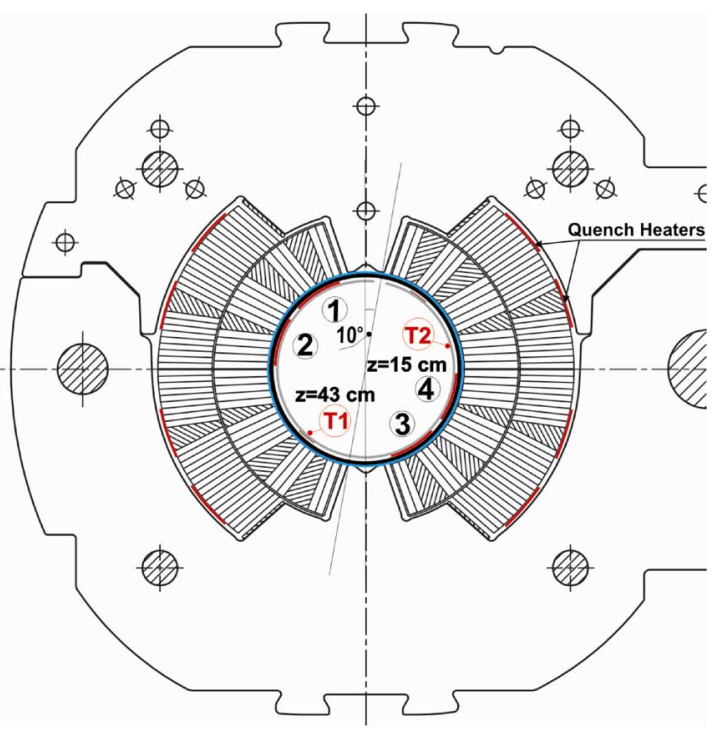

Fig. 8. Cross section of the main dipole magnet equipped with IHA. The heating strip and temperature probe azimuthal positions are shown.

at the end of the support tube (cf. Fig. 8 and Fig. 9). The temperature of the magnet helium bath has been measured by means of the temperature probe installed inside the magnet collar.

The experimental setup consisting of IHA and DC power supplies, enables to provoke quench for magnet currents between a few hundred amperes up to ultimate magnet current of $12850 \mathrm{~A}$. Before reaching the magnet coil and provoking a quench the heat is transferred throughout the heating strip insulation, the cold bore tube, the cold bore tube insulation and finally the helium channel, which is surrounding the cold bore. In Fig. 7 a photo of IHA, ready to be installed inside the magnet is presented.

Fig. 8 and Fig. 9 show chosen positions of the heating strips and temperature probe inside the MB and MQ magnets.

The numerical simulations and measurement results obtained on the MB and MQ magnets are shown in Fig. 10 and Fig. 11. The difference between measured and simulated values is shown in Fig. 12.

For both investigated magnets (main dipole MB and main focusing quadrupole MQ) the largest difference between measurements and simulations was found to be of the order of $70 \%$ for low MB magnet current, whereas the majority of the differences did not exceed $40 \%$.

The results of the simulation for nominal current with expected beam loss profile (Fig. 5 and Fig. 6) show that for the radial and for Gaussian beam loss profiles the quench level for 


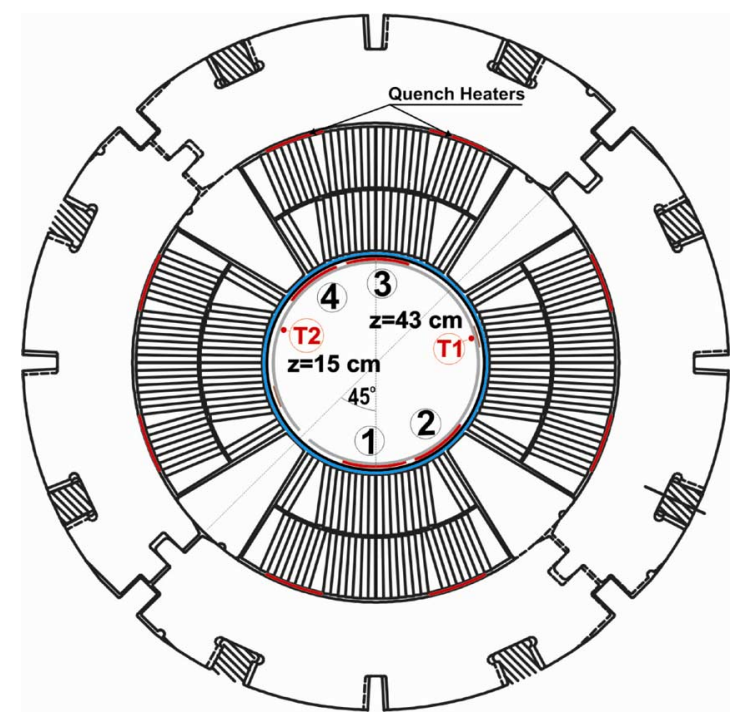

Fig. 9. Cross section of the main focusing quadrupole magnet equipped with IHA. The heating strip and temperature probe azimuthal positions are shown.

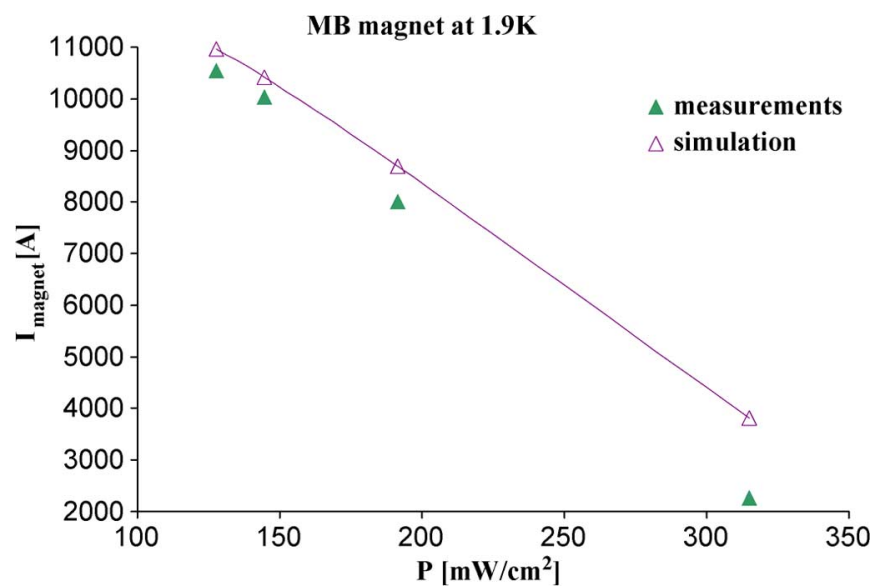

Fig. 10. Results of the measurements (markers) and simulation (solid line) for the MB magnet at $1.9 \mathrm{~K}$. The power dissipated in the heating strips is shown on $\mathrm{x}$-axis and corresponding magnet quench current is depicted on $\mathrm{y}$-axis.

MB dipole magnets is of the order of $12 \mathrm{~mW} / \mathrm{cm}^{3}$ and 17 $\mathrm{mW} / \mathrm{cm}^{3}$, respectively. The corresponding values for MQ focusing quadrupole magnets are $17 \mathrm{~mW} / \mathrm{cm}^{3}$ and $23 \mathrm{~mW} / \mathrm{cm}^{3}$ respectively.

\section{CONCLUSION}

The knowledge of the quench levels will allow setting appropriate initial threshold values for the beam loss monitor system. It is expected to increase the operational efficiency of the LHC. The measurements performed at $1.9 \mathrm{~K}$ and relevant numerical simulations show a relative difference less than $40 \%$ for most of the measurements. This precision is regarded as sufficient for the quench level knowledge [3]. The developed Network Model are used for the quench limit calculation of all LHC magnet types working both at $1.9 \mathrm{~K}$ and at $4.5 \mathrm{~K}$.

\section{ACKNOWLEDGMENT}

The authors thank F. Boisier, G. D'Angelo, J. Halik, P.Legrand and R. Mompo for their help with this work.

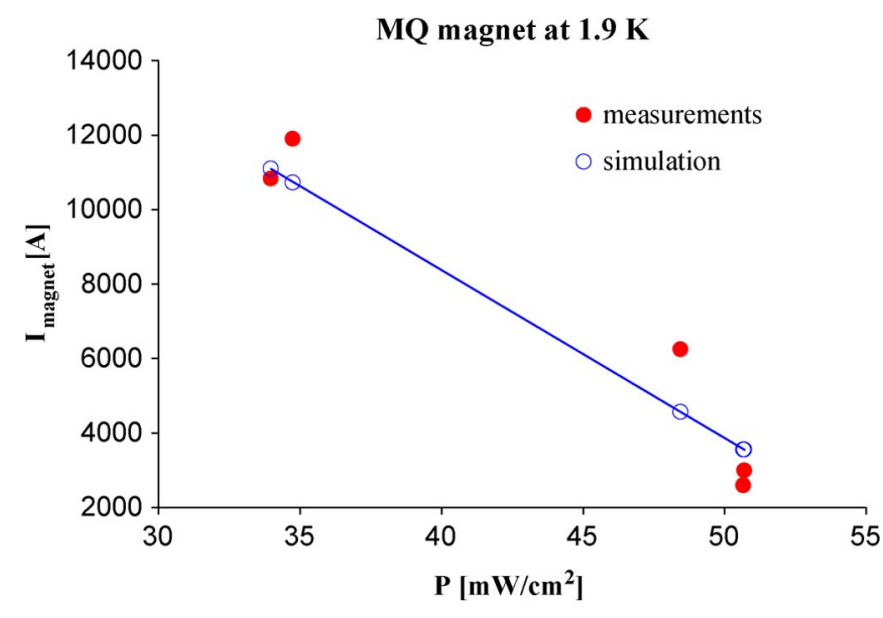

Fig. 11. Results of the measurements (markers) and simulation (solid line) for the MQ magnet at $1.9 \mathrm{~K}$. The power dissipated in the heating strips is shown on $\mathrm{x}$-axis and corresponding magnet quench current is depicted on $\mathrm{y}$-axis.

\section{MB and MQ magnet at 1.9K}

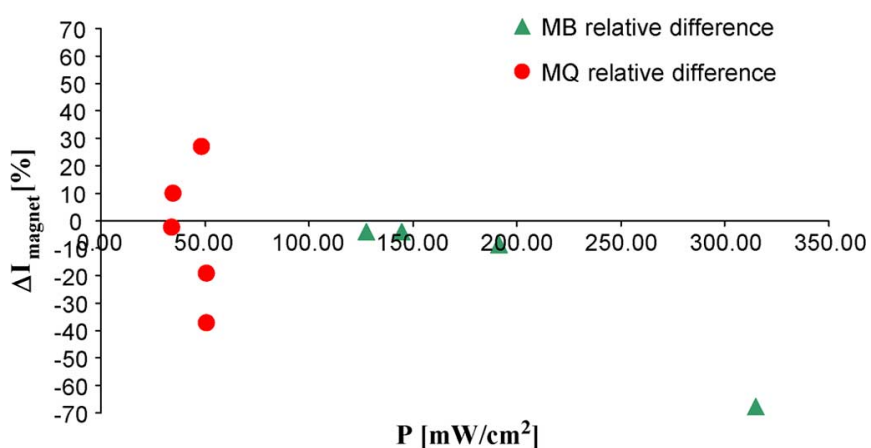

Fig. 12. Relative difference between measurements and simulations for MB magnet. The power dissipated in the quench heaters is shown on $\mathrm{x}$-axis and relative difference between measurements and simulations is depicted on $y$-axis

\section{REFERENCES}

[1] LHC Design Report, The LHC Main Ring CERN-2004-003, vol. 1.

[2] R. Schmidt et al., Beam Loss Scenarios and Strategies for Machine Protection at the LHC CERN, LHC Project Report 665, 2003.

[3] J. B. Jeanneret et al., On the Measurement of the Beam Losses in the LHC Rings CERN, LHC-BLM-ES-0001, 2004.

[4] B. Dehning et al., Design of the Beam Loss Monitoring System for the LHC Ring CERN, LHC Project report 781, 2004.

[5] E. B. Holzer et al., Generation of 1.5 Million Beam Loss Threshold Values CERN, LHC Project Report 1158, 2008.

[6] F. Bordry et al., Machine Protection for the LHC: Architecture of the Beam and Powering Interlock Systems CERN, LHC Project Report 521, 2001.

[7] D. Bocian et al., "Modelling of quench limit for steady state heat deposits in LHC magnets," IEEE Appl. Trans. Supercond., vol. 18, no. 2, pp. 112-115, Jun. 2008.

[8] L. Sarchiapone, private communication.

[9] J. B. Jeanneret et al., Quench Levels and Transient Beam Losses in LHC Magnets CERN, LHC Project Report 44, 1996.

[10] Cryosoft, France, 2001.

[11] S. W. Van Sciver, Helium Cryogenics. New York: Plenum Press, 1986.

[12] C. Schmidt, Review of Steady State and Transient Heat Transfer in Nucleate Boiling Helium I. Saclay, France, International Institute of Refrigeration: Commission A 1/2-Saclay, 1981, pp. 17-31.

[13] M. Nishi et al., "Boiling helium heat transfer characteristics in narrow cooling channel," IEEE Trans. Magn., vol. MAG-19, no. 3, pp. 390-393, 1983. 